\title{
Acknowledgment to Reviewers of Non-Coding RNA in 2021
}

\author{
Non-Coding RNA Editorial Office
}

MDPI AG, St. Alban-Anlage 66, 4052 Basel, Switzerland

Citation: Non-Coding RNA Editorial Office. Acknowledgment to Reviewers of Non-Coding RNA in 2021. Non-coding RNA 2022, 8, 14. https://doi.org/10.3390/ncrna8010014

Published: 29 January 2022

Publisher's Note: MDPI stays neutral with regard to jurisdictional claims in published maps and institutional affiliations.

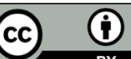

Copyright: (C) 2022 by the author. Licensee MDPI, Basel, Switzerland. This article is an open access article distributed under the terms and conditions of the Creative Commons Attribution (CC BY) license (https://creativecommons.org/licenses/by/4.0/).
Rigorous peer-reviews are the basis of high-quality academic publishing. Thanks to the great efforts of our reviewers, Non-Coding RNA was able to maintain its standards for the high quality of its published papers. Thanks to the contribution of our reviewers, in 2021, the median time to first decision was 15 days and the median time to publication was 37 days. The editors would like to extend their gratitude and recognition to the following reviewers for their precious time and dedication, regardless of whether the papers they reviewed were finally published:

$\begin{array}{ll}\text { Abhishek Dey } & \text { David C. Zappulla } \\ \text { Alan Dombkowski } & \text { David Leavesley } \\ \text { Alessandro Sammarco } & \text { Deepanjan Paul } \\ \text { Alex De Lencastre } & \text { Edoardo Bertolini } \\ \text { Alexey Nikulin } & \text { Elena López-Jiménez } \\ \text { Alfonso M. Cayota } & \text { Erica Scott } \\ \text { André Gerber } & \text { Estanis Navarro } \\ \text { Andrea Caporali } & \text { Esteban Orellana } \\ \text { Angel Vizoso-Vázquez } & \text { Federico Dajas-Bailador } \\ \text { Anisha Gupta } & \text { Francisco J. Enguita } \\ \text { Anna Riester } & \text { Franck Martin } \\ \text { Antoine M. Dujon } & \text { Gaetano Santulli } \\ \text { Antonio Musio } & \text { Gianpiero Di Leva } \\ \text { Aria Baniahmad } & \text { Giulia Matacchione } \\ \text { Arijita Sarkar } & \text { Giuseppe Palmieri } \\ \text { Arjen E. Van't Hof } & \text { Giuseppina E. Grieco } \\ \text { Arturo López Castel } & \text { Grasiele Sausen } \\ \text { Assam El-Osta } & \text { Gunter Meister } \\ \text { Babu V. Sajesh } & \text { Helen Vincent } \\ \text { Bei Cheng } & \text { Henrik Nielsen } \\ \text { Bodhisattwa Banerjee } & \text { Hiroji Aiba } \\ \text { Byron Baron } & \text { Hisashi Mera } \\ \text { Cameron Bracken } & \text { Hua-Sheng Chiu } \\ \text { Cecilia Battistelli } & \text { Hui Li } \\ \text { Celia Fernández Rubio } & \text { Ilaria Guerriero } \\ \text { Cesar Lopez-Camarillo } & \text { Izabella Slezak-Prochazka } \\ \text { Charles Bou-Nader } & \text { Jan Novák } \\ \text { Christine Happel } & \text { Jarmila Hnilicová } \\ \text { Christopher Hellen } & \text { Jens Hahne } \\ \text { Christos K. Kontos } & \text { Jianhua Wang } \\ \text { Christos Papaneophytou } & \text { Jiao Jiao Li } \\ \text { Claudia Kutter } & \text { Jijun Huang } \\ \text { Cristian Taccioli } & \text { Joanna Sztuba-Solinska } \\ \text { Daniela Gradia } & \text { Joanna Williams } \\ & \\ & \end{array}$


Joe Chihade

José Andrade

Juan José González-Plaza

Katherine McJunkin

Kathrin Tyryshkin

Kausik Chakrabarti

Lasse Lindahl

Lei Shi

Leonard Lipovich

Letizia Pitto

Lin Huang

Lorenzo Farina

Luca Agnelli

Luca Lo Piccolo

Lucia Natarelli

Luke Selth

Mai Hazekawa

Marco Gaviraghi

Margarida Gama-Carvalho

Maria Duhagon

Maria Elizabeth Rossi Da Silva

Maria Hondele

Maria Sundvall

Marina Cristodero

Martin Sztacho

Martina Kirchner

Martina Rossi

Matthias S. Leisegang

Matus Sykora

Md. Motiar Rahman

Michael Tellier

Michele De Bortoli

Mohammed L. Abba

Munekazu Yamakuchi

Natalia Simionescu

Nicholas Delihas

Nicolas Casadei

Nicoletta Bianchi

Nithyananda Thorenoor

Oliver Mühlemann

Ovidiu Sirbu
Pablo Smircich

Panagiotis Alexiou

Paola Ostano

Patricia Mirella Scardua

Pavel Ivanov

Peter Bai

Praveen Arany

Przemysław Nuc

Qinyu Sun

Rajneesh Srivastava

Robert Weinzierl

Rocio T. Martinez Nunez

Roland Klassen

Rui Fu

Russell Hamilton

Ryuya Fukunaga

Sabyasachi Dash

Samuel Pushparaj Robert Jeyasingh

Samuela Pasquali

Satya Kota

Sébastien Pfeffer

Shahrokh Ghobadloo

Shizuka Uchida

Shu-Ling Tzeng

Silvia Lee

Simon Conn

Simon Rayner

Simona Zaami

Stefan Ernst Seemann

Stefano Cagnin

Stephan Bernhart

Stephan Hamperl

Suba Rajendren

Sumit Mukherjee

Tadashi Yoshida

Tanja Kunej

Vasiliki Tsigkou

Xucheng Hou

York Marahrens

Yoshihiro Taguchi 